\title{
Manufatura Avançada para Produção Agrícola usando Inteligência Artificial e IoT
}

\author{
Wesley R. da Silva* Jefferson C. G. de Almeida* \\ Emerson F. de Oliveira* Egídio R. Neto* Carlos A. Ynoguti* \\ João P. C. Henriques * \\ * Instituto Nacional de Telecomunicações,Inatel, MG, (e-mail: \\ wesleyreis@gec.inatel.br, jeffersonalmeida@gec.inatel.br, \\ emerson.oliveira@gec.inatel.br, egidio.neto@inatel.br, \\ ynoguti@inatel.br,joao.paulo@inatel.br).
}

\begin{abstract}
Advanced manufacturing in the context of the fourth industrial revolution is characterized by the integration of technologies to make productive processes autonomous and intelligent. This revolution brought improvements not only for the industry field: The agriculture for instance, is one of the most impacted areas by this technological revolution. Therefore, this project proposal is to demonstrate the interaction between the Artificial Intelligence - AI for voice recognition with Internet of Things - IoT protocols to control a hardware system and provide the agricultural production automation. The proposed system can be handled by user and consists in a garden, where all planting and cultivation processes are autonomous, allowing people to harvest in large urban centers, where time and space are limited.

Resumo: A manufatura avançada refere-se à $4^{\mathrm{a}}$ Revolução Industrial, caracterizada pela integração de tecnologias a fim de interligar os processos produtivos tornando-os mais autônomos e inteligentes. Esta revolução não traz melhorias somente para as indústrias, a agricultura por exemplo é um dos setores que estão recebendo uma grande contribuição tecnológica. No presente contexto, este trabalho tem como finalidade demostrar a interação da Inteligência Artificial - IA pelo reconhecimento de voz com protocolos de dados Internet das Coisas - IoT controlando um sistema de hardware, visando automatizar a produção de alimentos. O sistema proposto consiste basicamente em uma horta em que o processo de plantar e cultivar alimentos é autônomo, permitindo a produção em grandes centros urbanos, visto que tempo e espaço são recursos limitados.
\end{abstract}

Keywords: Smart Control; Embedded System; IoT; Artificial Intelligence; Autonomous Agriculture.

Palavras-chaves: Controle Inteligente; Sistema Embarcado; IoT; Inteligência Artificial; Agricultura Autônoma.

\section{INTRODUÇÃO}

A produção de alimentos dentro dos limites das cidades (agricultura urbana), está ganhando atenção crescente como uma componente importante para uma economia sustentável, saúde pública e desenvolvimento comunitário, entre outros fatores (Kirkpatrick and Davison, 2018).

Em países ricos, planta-se em casa por hobby ou para poder ter acesso a alimentos saudáveis, sem o uso de agrotóxicos (Dobernig and Stagl, 2015), enquanto que em países com menos recursos ou em crise econômica, plantase para sobreviver (Round et al., 2010).

Ainda, a sociedade moderna impôs um ritmo de vida bastante acelerado, principalmente nos grandes centros urbanos. Cuidar de uma horta pode se tornar um desafio: é necessário conhecer as necessidades e cuidados para cada espécie, irrigá-las periodicamente, etc. Um sistema que cuidasse automaticamente destas tarefas, otimizando as ações para cada tipo específico de planta, seria de grande ajuda.

Novas tecnologias podem potencialmente trazer benefícios às pessoas. Recentemente, a Internet das Coisas (IoT Internet of Things) começou a exercer um papel maior em nossas vidas diárias, estendendo nossas percepções e habilidades de modificar o ambiente ao nosso redor. O campo da agro-indústria aplica esta tecnologia, junto com técnicas de IA - Inteligência Artificial, tanto para diagnóstico como para controle (Muangprathub et al., 2019). Alguns sistemas já foram propostos, para irrigação (Li-fang, 2012; Tripathy et al., 2015; Divani et al., 2016) e semeadura automáticos (Jayakrishna et al., 2018).

Neste trabalho é proposto um sistema para automatizar o plantio e cultivo de alimentos em pequena escala, usando os conceitos de IoT, assistentes virtuais inteligentes - AVI, sistemas embarcados e controles inteligentes. Ao sistema proposto deu-se o nome de Cultbot, uma aglutinação das palavras Cultivator e Robot. 


\section{AGRICULTURA DE PRECISÃO}

Se popularizou como uma técnica de tratar a agricultura em busca de um alto rendimento levando em conta localização, fertilidade do solo entre outros fatores importantes no cultivo (Lamparelli, 2005). Agricultura de precisão, é o sistema de produção adotado por agricultores de países de tecnologia avançada que surgiu como um sistema de gerenciamento de informações e que teve seu crescimento potencializado a partir de avanços da tecnologia de referenciamento e posicionamento, como o GPS e de tecnologias de sensoriamento remoto (Bernardi et al., 2014).

Agricultura de precisão engloba o uso de tecnologias atuais com uma agricultura industrial, para o manejo de solo, insumos e culturas, de modo à adequar as variações espaciais e temporais em fatores que afetam a produtividade das mesmas. O conjunto de tecnologias é um sistema composto de sensores e atuadores que são integrados para obter controle de produção, tentando igualar todos os recursos, ou seja, insumos que são utilizados em toda área de cultivo (Davis et al., 1998).

Para Coelho et al. (2004), existem sete princípios da agricultura de precisão, destacando-se: sistema de posicionamento (GPS), sistema de informação geográfica (SIG), análise de solos, detecção remota, sistemas de monitorização ambiental e da produtividade, aplicações diferenciadas (VTR - Variable rate technology), aspectos econômicos da agricultura de precisão. A Figura 1 apresenta os 3 tópicos bases utilizados no desenvolvimento do projeto.

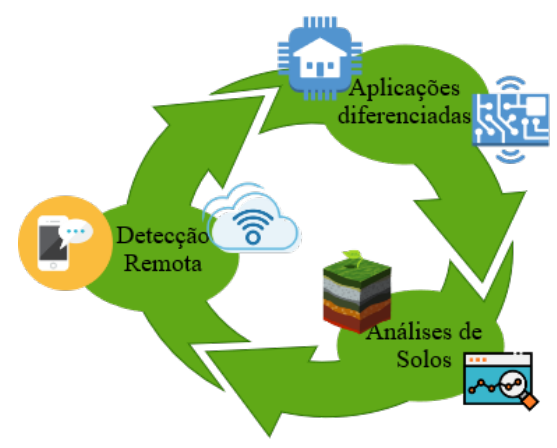

Figura 1. Princípios base da agricultura de precisão utilizados

Dado o fato de automatização e visualização de dados se faz necessário o uso de conceitos de manufatura avançada que será explicado na próxima sessão.

\subsection{Manufatura Avançada}

Manufatura avançada é um processo de implantação, concepção e desenvolvimento de tecnologias que facilitam o processo e produção, tornado as indústrias mais inteligentes. Também vem agregada com a $4^{\circ}$ revolução industrial que envolve protocolos de IoT - Internet das Coisas e grandes volumes de dados (Big data) que podem ser cruzados para tornar cada vez mais efetivo o processo industrial (Arbix et al., 2017).

Segundo Kupfer (2016) "Manufatura Avançada ou Indústria 4.0 nada mais são do que um elenco de técnicas que dependem do uso coordenado de informação, automação, computação, software, sensoriamento e conexão em rede", permitindo assim que as indústrias alcancem níveis nunca imaginados de customização, organização, autonomia e otimização.

Na manufatura avançada se faz necessário o uso de protocolos específicos de internet das coisas para conexão e comunicação entre dispositivos que coletam informações importantes do sistema, para melhor entendimento será detalhado na próxima sessão.

\subsection{Protocolos}

Para transmissão dos dados foi utilizado o protocolo MQTT - Message Queuing Telemetry Transport que foi desenvolvido pela IBM, seu funcionamento é baseado em um servidor e um cliente, Raspberry e smartphone respectivamente. A comunicação é feita através de publicações (publish) e inscrições (subscribe) controladas pelo broker (Desai, 2015).

A troca de dados é semelhante a um sistema de fórum web. O cliente, ao se conectar ao broker, deve se inscrever em um ou mais tópicos para enviar ou receber informações. Os dados publicados a um tópico são enviados a todos os dispositivos inscritos ao mesmo. A Figura 2 apresenta essa comunicação (Waher, 2015).

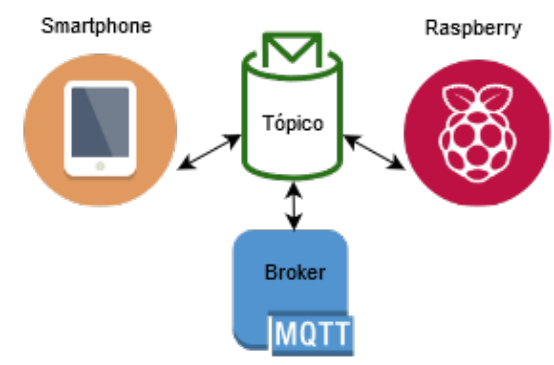

Figura 2. Protocolo MQTT

HTTP - Hypertext Transfer Protocol é um protocolo para hipermídia baseado em requisições e respostas a um servidor. O cliente solicita um determinado recurso (resquest) a um ponto de acesso (endereço URL - Uniform Resource Locator), enviando um pacote que contém cabeçalho, que e em alguns casos podem conter dados. O servidor recebe a requisição, processa e envia a resposta (response) que contém o recurso solicitado ou apenas o cabeçalho (BernersLee et al., 1996).

\subsection{Internet das Coisas}

Internet das Coisas vem da ideia de embutir sensores a equipamentos, para assim gerar dados que podem servir de alimentação para outra plataforma inteligente a fim de tomar decisões automáticas sem interferência humana (Gubbi et al., 2013).

A internet das coisas pode conectar vários objetos do cotidiano a rede, utilizado protocolos como: MQTT e HTTP. Todos estes dispositivos comunicam entre si trocando informações relevantes para a execução de alguma tarefa. Os dados coletados são enviados para nuvem que podem ser acessados com apenas uma conexão à internet, permitindo 
o controle remoto de todo o sistema (Shenoy and Pingle, 2016).

\subsection{Inteligência Artificial e Controle Inteligente de Dados}

Inteligência Artificial é uma tecnologia que possibilita que máquinas aprendam com dados que são inseridos em sua base de informações, através desse processo elas tendem a deixar o procedimento cada vez certeiro sem a interferência humana na análise (Teixeira, 2014). Quando aplicada no reconhecimento de voz por exemplo, é possível observar um melhor entendimento a medida que os comandos são executados.

Controle inteligente de informação é a transformação de dados brutos em informações base para tomada de decisões no sistema. Para que seja possível tal processo, o dado bruto deve passar por tratamentos a fim de correlatar dados inicialmente independentes tornando-os informações organizadas e confiáveis. Tecnologias como BigData, Data Science e IA são utilizadas nesse processo (Costa, 2006).

\section{SISTEMA PROPOSTO}

O sistema tem como base o funcionamento de uma impressora 3D, todo o conjunto foi montado em cima de um caixote de madeira que atua como horta de pequeno porte. O equipamento possui uma estrutura mecânica formada por barras de perfil alumínio e motores que permitem sua movimentação, a fim de mostrar e validar todo funcionamento dos recursos implementados.

A Cultbot é composta por um hardware que fica responsável pelo controle dos periféricos e outro pelo controle de informações e comunicação com a nuvem de dados. O hardware responsável pelo processamento é a Raspberry que por sua vez está concentrado o middleware que gerencia as informações e provê todo acesso a qualquer dado com ou sem conexão com a Internet. A Figura 3 mostra o diagrama completo do sistema.

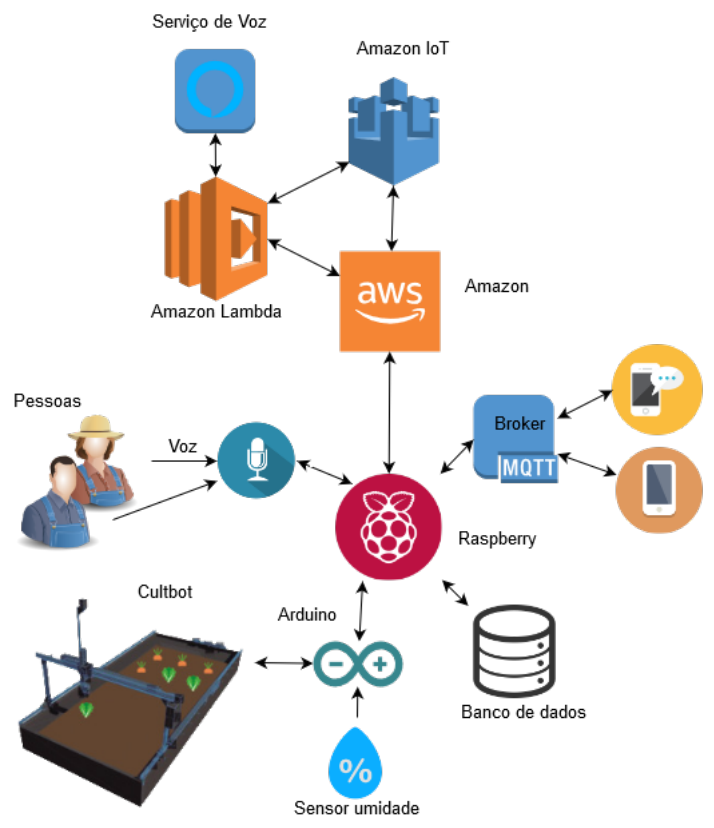

Figura 3. Diagrama do sistema
O aplicativo foi desenvolvido para tornar possível a interação com o sistema mesmo distante, em qualquer lugar que possua conexão com a internet ou localmente sem conexão com a internet.

Após o recebimento das instruções o sistema embarcado realiza uma série de rotinas, que executam os movimentos ou fazem a leitura dos sensores, disponibilizando os dados no aplicativo Android ou para a assistente virtual Alexa.

A interação com a Cultbot pode ser realizada de duas maneiras diferentes, sendo elas: um sistema totalmente autônomo com rotinas para coleta de dados e execução de tarefas como, plantar, medir umidade e regar; ou um sistema com total controle do usuário, sendo por reconhecimento de voz utilizando a AVI ou pelo aplicativo mobile para todas as funções citadas.

\subsection{Mecânica}

Para construção do projeto se fez necessário uma estrutura que pudesse ser facilmente operada e de tamanho variável, de forma que pudesse ser inserida dentro de residências. A Figura 4 apresenta o sistema proposto.

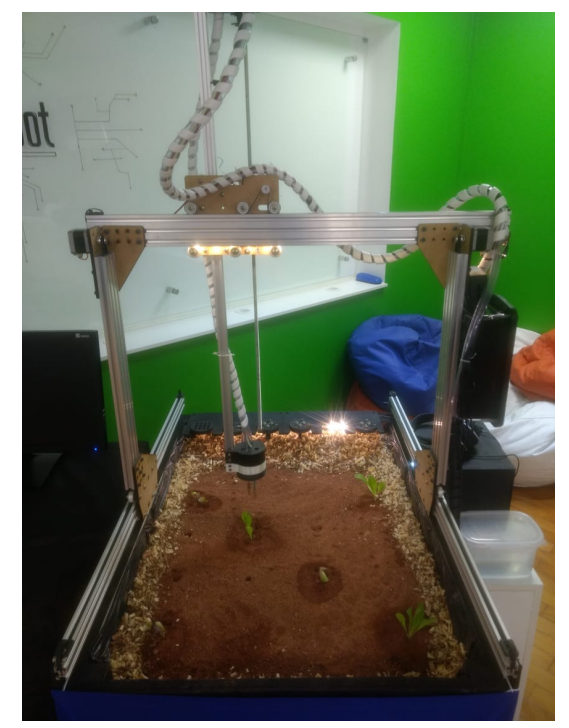

Figura 4. Cultbot

A mecânica foi desenvolvida na forma de um sistema CNC - comando numérico computadorizado de três eixos x, y e z. A estrutura foi construída com perfil de alumínio, peças impressas em impressora 3D e cortadas a laser em MDF Medium Density Fiberboard.

O design foi baseado em um projeto open-source, Farmbot (2018), que disponibiliza algumas ferramentas que foram modificadas e usadas na construção da estrutura. A Cultbot dispõe de diversas ferramentas para manuseio da horta: um semeador, um regador e um sensor de umidade como mostrado na Figura 5. Para a troca dinâmica desses equipamentos foram utilizados ímãs que possibilitam o encaixe e desencaixe de maneira simples com apenas a aproximação do bico da CNC.

\subsection{Inteligência do Sistema}

Com intuito de automatizar o sistema foram utilizados recursos da Amazon (2018), empresa que disponibiliza ser- 


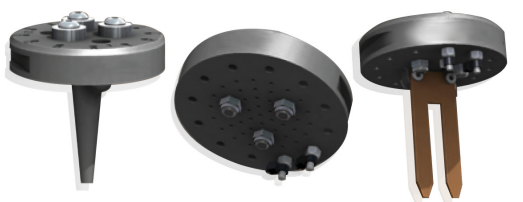

Figura 5. Ferramentas modulares

viços de computação em nuvem fornecendo vários módulos dentro do Amazon Web Services - AWS, alguns desses módulos foram integrados juntos com a assistente virtual Alexa.

A assistente virtual utiliza o sistema de comunicação feito pelo reconhecimento de voz, que é processado por uma IA a fim de facilitar a interação com o usuário sendo extremamente precisa no entendimento da ação requisitada, evitando assim varios clicks para completar uma tarefa. A assitente também possibilita incluir diversos comandos personalizados, devido ao serviço Alexa Skill, que permite criar habilidades possibilitando à Alexa aprender responder (Developer, 2019).

Todos os serviços AWS utilizados foram interligados, possibilitando todo o tratamento de eventos que são enviados para um servidor cuja as informações são processadas, o AWS Lambda. Após a resposta do servidor a informação é enviada ao modulo que realiza a comunicação com todos os dispositivos integrados na plataforma online (Kurniawan, 2018).

\subsection{Comunicação}

Um dos protocolos utilizados para comunicação foi MQTT, no sistema em questão é usado para realizar a comunicação entre o middleware, a Alexa e o aplicativo Android. Dados como umidade, posição das plantas, tipo de cada planta e quando foi plantada são enviados por este protocolo. A escolha deste protocolo se deve ao fato de que ele foi projetado para o baixo consumo de banda e plataformas no qual os requisitos de hardware são relativamente simples (Chen et al., 2014).

Outro protocolo utilizado foi o HTTP que está bastante presente em sistemas IoT. No sistema em questão o HTTP foi utilizado para enviar as informações sobre o atual estado do sistema para uma base de dados na AWS. A Alexa acessa essa base de dados e responde ao usuário com base nestas informações (Rodriguez, 2008).

\subsection{Middleware}

Realiza todas as conversões geradas pelo sistema de IA e pelo hardware. Foi idealizado e criado junto ao sistema embarcado para realizar a integração em ambos os sistemas, possibilitando que uma pessoa pudesse realizar o pedido a IA ou aplicativo mobile e o mesmo ser executado rapidamente. O middleware facilita a comunicação de entrada e saída de dados da para o sistema, transportando todos estes dados pelos protocolos de comunicações.

\subsection{Hardware}

O sistema é constituído por várias ferramentas que podem ser acoplados facilmente ao hardware, o processo de troca foi designado ao sistema embarcado onde se encontra a IA Alexa.

O hardware pode ser dividido em três partes: um microprocessador (BCM2836 - Raspberry), um microcontrolador (ATMega 2560 - Arduino Mega) e a estrutura de movimentação, sensoriamento e acionamento.

A estrutura é composta por uma bomba a vácuo, utilizada para coletar as sementes a serem plantadas; uma bomba de água, usada para regar as plantas; um sensor de umidade do solo e toda parte de movimentação, formada por motores de passo, correias e um fuso trapezoidal.

A lógica do sistema está no microprocessador, é nele que está o middleware em conjunto com o serviço da assistente virtual Alexa. As ações a serem realizadas são processadas pela Alexa e enviadas para middleware utilizando o protocolo MQTT, que verifica no banco de dados as informações a respeito da Cultbot e quebra a ação em pequenas instruções que são enviadas via comunicação serial para o microcontrolador.

O microcontrolador é responsável por receber instruções e executa-las. As instruções podem ser: acionar motores, acionar bomba a vácuo e fazer a leitura do sensor. A Figura 6 ilustra o relacionamento do microcontrolador com as demais partes.

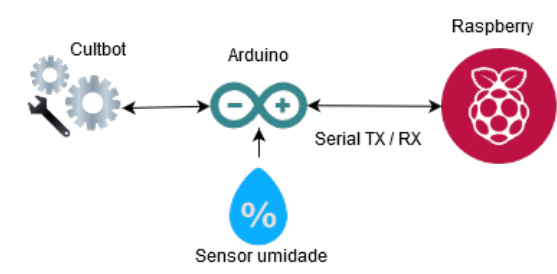

Figura 6. Diagrama de conexões com microcontolador

O controle de irrigação foi pensado para diminuir o desperdício de água. Para irrigar apenas o necessário foi utilizado um sensor de umidade que periodicamente coleta os dados de umidade das plantas, evitando assim o desperdício e possíveis doenças causadas pela má irrigação, já que o sistema irrigará na hora e na quantidade correta. Todas essas etapas de validação de dados e uso de informações são consideradas parte da agricultura de precisão, pois atuam na questão de recursos mínimos levando cada vez mais a eficiência no processo de plantio.

\subsection{Software}

Aplicativo Android desenvolvido pela equipe possui uma interface que permite o controle de toda a plantação mantendo registro das informações como: o dia do plantio, dia para colheita, umidade, e posição/tipo das plantas já existentes. No mesmo também é possível a execução das funções de todo o sistema como: regar, plantar e medir a umidade. Figura 7 apresenta a tela do app.

A interface de voz implantada no microprocessador possibilita a interação do usuário com a Alexa. Seu acionamento é realizado através da chamada do nome, em seguida é 


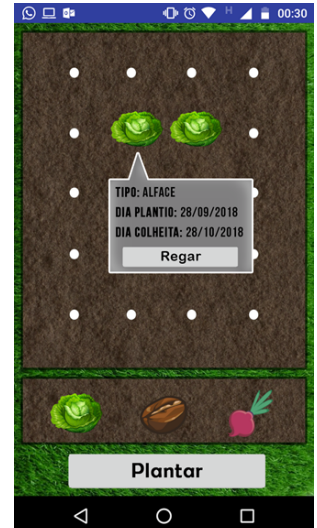

Figura 7. Aplicativo mobile

possível realizar algum pedido que foi programado previamente no servidor da Amazon e tratado no sistema do middleware. Com esta interface, o usuário também pode executar as mesmas funções que estão disponíveis no aplicativo Android, porém através de comandos de voz como: "Alexa, ask machine to plant coffee at position 1", "Alexa, ask machine to get humidity" entre outros.

\section{RESULTADOS}

A Cultbot trás uma diferença se comparada ao sistema da Farmbot, tal fator se concentra especialmente no reconhecimento de voz e execução de atividades sem a necessidade do manuseio manual do equipamento, permitindo que pessoas com idades ou condições físicas diversas utilizem o sistema.

Todo controle pode ser feito pelo reconhecimento de voz ou pelo aplicativo de celular. Além do controle, tem-se em mãos dados de todos os processos em tempo real, podendo ser consultados e utilizados para tomadas de decisões a respeito da atividade a ser realizada na horta, tornando assim o processo de manuseio ajustável, caso ocorra uma interferência biológica/climática. No sistema montado é possível setar os tipos de plantas e as distâncias que cada planta será plantada, otimizando assim o espaço para plantio.

O ambiente onde o sistema foi inserido foi montado de forma a ser semelhante a um ambiente doméstico, utilizando como meio de conexão uma rede sem fio compartilhada com link de $10 \mathrm{Mbps}$. Dentro deste cenário algumas medidas foram coletadas com intuito de quantizar o tempo de resposta do sistema dado o envio de um comando. Para a comunicação através do aplicativo utilizando o protocolo MQTT, verificou-se uma média de tempo de resposta de 1.88 segundos, a Figura 8 apresenta as medidas realizadas.

Para o tempo de resposta a assistente virtual temos uma media de 3.38 segundos para uma resposta efetiva de qual ação executar, pois toda informação percorre os módulos em nuvem da Amazon, a Figura 9 apresenta as medidas realizadas.

O método de sucção, utilizado para pegar as sementes, se mostrou pouco eficiente no controle do número de sementes plantadas e na manipulação diferentes tipos. Isso se deve a diferença de tamanho, peso e forma das sementes utilizadas.

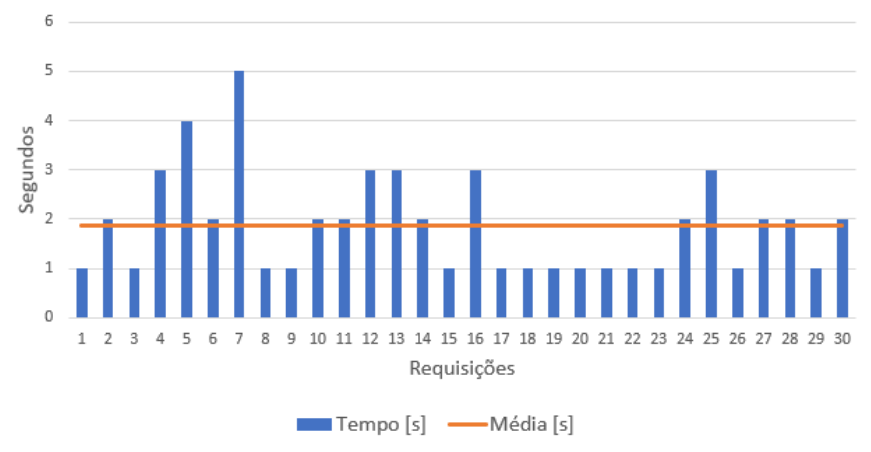

Figura 8. Tempo resposta aplicativo Android

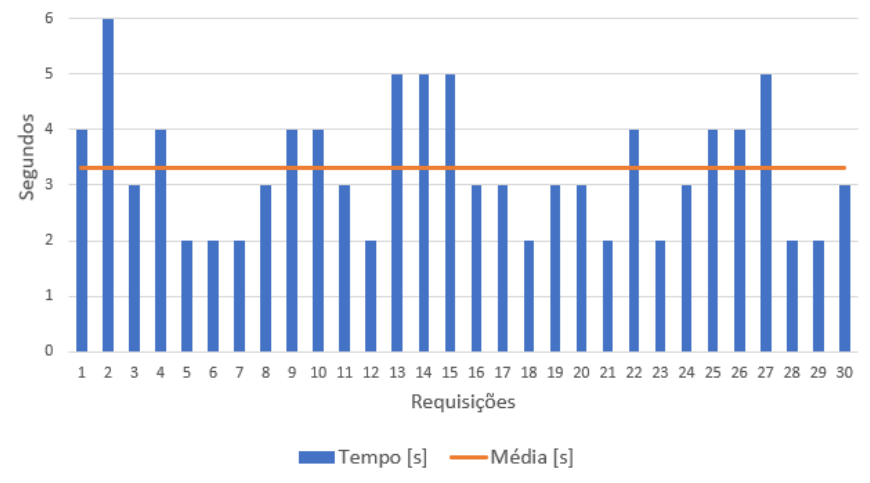

Figura 9. Tempo resposta assistente virtual

Contudo, mecânica utilizada realiza as tarefas de plantar, regar e monitorar a umidade do solo de forma semelhante a Farmbot. Esse resultado se mostrou satisfatório tendo em vista que a mecânica foi construída utilizando peças alternativas impressas em impressoras $3 \mathrm{D}$ ou cortadas a laser em MDF a fim reduzindo o custo na construção do projeto.

\section{CONCLUSÃO}

Neste trabalho é apresentado um sistema para cultivo automático para uso doméstico no qual foram utilizados diversas tecnologias relacionadas a agricultura de precisão e industria 4.0, que trazem consigo diversos fatores de melhorias para a automatização que podem ser aplicados para um cultivo cada vez mais saudável. O desempenho desse sistema atende as expectativas para o qual foi projetado executando as tarefas de plantar, medir a umidade do solo e regar os alimentos de forma inteligente e autônoma. Os tempos de resposta analisados no sistema são aceitáveis visando aplicações residenciais, na qual a velocidade na execução das tarefas não é prioridade e sim a automatização de todo o processo.

Um problema notado foi a incompatibilidade do aplicativo, optar por desenvolver em Android limitou sua utilização por outros celulares. Outro ponto negativo é a número de idiomas disponíveis para a Alexa, o que dificulta sua usabilidade por parte do público geral.

Como próximos passos pretende-se: avaliar os impactos do controle inteligente da irrigação na produtividade dos alimentos e desenvolver um aplicativo híbrido permitindo 
assim a sua utilização por uma gama muito maior de dispositivos.

\section{REFERÊNCIAS}

Amazon (2018). Amazon Web Services. URL https:// aws. amazon. com/pt/.

Arbix, G., Salerno, M.S., Zancul, E., Amaral, G., and Lins, L.M. (2017). O Brasil e a nova onda de manufatura avançada: O que aprender com Alemanha, China e Estados Unidos. Novos estudos CEBRAP, 36, $29-49$. URL http://www.scielo.br/scielo.php?script= sci_arttext\&pid=S0101-33002017000300029\&nrm= iso.

Bernardi, A.d.C., Naime, J.d.M., RESENDE, A.d., Inamasu, R., and BASSOI, L. (2014). Agricultura de precisão: resultados de um novo olhar. Embrapa Instrumentação-Livro técnico (INFOTECA-E).

Berners-Lee, T., Fielding, R., and Frystyk, H. (1996). Hypertext transfer protocol-http/1.0. Technical report.

Chen, W.J., Gupta, R., Lampkin, V., Robertson, D.M., Subrahmanyam, N., et al. (2014). Responsive Mobile User Experience Using MQTT and IBM MessageSight. IBM Redbooks.

Coelho, J.C., Silva, L.M., Tristan, M., Neto, M.d.C., and Pinto, P.A. (2004). Agricultura de precisão. Prefácio, Lisboa.

Costa, H.R.d.N. (2006). Aplicação de técnicas de inteligência artificial em processos de fabricação de vidro. Ph.D. thesis, Universidade de São Paulo.

Davis, G., Casady, W.W., and Massey, R.E. (1998). Precision agriculture: An introduction. Extension publications (MU).

Desai, P. (2015). Python programming for Arduino. Packt Publishing Ltd.

Developer, A. (2019). Alexa Voice Service Overview. URL https://developer.amazon.com/ alexa-voice-service.

Divani, D., Patil, P., and Punjabi, S.K. (2016). Automated plant watering system. In 2016 International Conference on Computation of Power, Energy Information and Commuincation (ICCPEIC), 180-182. doi:10.1109/ ICCPEIC.2016.7557245.

Dobernig, K. and Stagl, S. (2015). Growing a lifestyle movement? exploring identity-work and lifestyle politics in urban food cultivation. International Journal of Consumer Studies, 39(5), 452-458. doi:10.1111/ ijcs.12222. URL https://onlinelibrary.wiley.com/ doi/abs/10.1111/ijcs.12222.

Farmbot (2018). Home Page. URL https://farm.bot/.

Gubbi, J., Buyya, R., Marusic, S., and Palaniswami, M. (2013). Internet of things (iot): A vision, architectural elements, and future directions. Future generation computer systems, 29(7), 1645-1660.

Jayakrishna, P., Reddy, M.S., Sai, N.J., Susheel, N., and Peeyush, K. (2018). Autonomous seed sowing agricultural robot. In 2018 International Conference on Advances in Computing, Communications and Informatics (ICACCI), 2332-2336. IEEE.

Kirkpatrick, J.B. and Davison, A. (2018). Home-grown: Gardens, practices and motivations in urban domestic vegetable production. Landscape and Urban Planning, 170, 24 - 33. doi:https://doi.org/10.1016/j.landurbplan.
2017.09.023. URL http://www.sciencedirect.com/ science/article/pii/S0169204617302542.

Kupfer, D. (2016). Indústria 4.0 brasil. Valor Econômico, 8.

Kurniawan, A. (2018). Learning AWS IoT: Effectively manage connected devices on the AWS cloud using services such as AWS Greengrass, AWS button, predictive analytics and machine learning. Packt Publishing Ltd.

Lamparelli, R.A.C. (2005). Agricultura de precisão. URL http://www.agencia.cnptia.embrapa.br/ gestor/cana-de-acucar/arvore/CONTAG01_72_ 711200516719.html.

Li-fang, T. (2012). Application of autocontrol technology in water-saving garden irrigation. In 2012 International Conference on Computer Science and Information Processing (CSIP), 1311-1314. IEEE.

Muangprathub, J., Boonnam, N., Kajornkasirat, S., Lekbangpong, N., Wanichsombat, A., and Nillaor, P. (2019). Iot and agriculture data analysis for smart farm. Computers and Electronics in Agriculture, 156, 467 - 474. doi:https://doi.org/10.1016/j.compag.2018.12. 011. URL http://www.sciencedirect.com/science/ article/pii/S0168169918308913.

Rodriguez, A. (2008). Restful web services: The basics. IBM developer Works, 33, 18.

Round, J., Williams, C., and Rodgers, P. (2010). The role of domestic food production in everyday life in post-soviet ukraine. Annals of the Association of American Geographers, 100(5), 1197-1211. doi:10.1080/ 00045608.2010.520214. URL https://doi.org/10. 1080/00045608.2010.520214.

Shenoy, J. and Pingle, Y. (2016). Iot in agriculture. In 2016 3rd International Conference on Computing for Sustainable Global Development (INDIACom), 14561458. IEEE.

Teixeira, J.F. (2014). Inteligência artificial. Pia Sociedade de São Paulo-Editora Paulus.

Tripathy, A.K., Vichare, A., Pereira, R.R., Pereira, V.D., and Rodrigues, J.A. (2015). Open source hardware based automated gardening system using low-cost soil moisture sensor. In 2015 International Conference on Technologies for Sustainable Development (ICTSD), 16. doi:10.1109/ICTSD.2015.7095915.

Waher, P. (2015). Learning internet of things. Packt Publishing Ltd. 